\title{
A Possible Stellar Interaction with NGC 246
}

\author{
Grant J. Miller ${ }^{1,2}$ \\ ${ }^{1}$ Mount Laguna Observatory, San Diego State University; \\ ${ }^{2}$ Department of Astronomy, Southwestern College
}

The planetary nebula NGC 246 has a high degree of symmetry about its central star, and can be described most graphically as a filled disk. Direct images of NGC 246 reveal a relatively homogeneous distribution of gases, with an unusual morphological feature that appears to contain less of the nebular material. At the center of this roughly circular feature, in the southeast quadrant of the nebula, there is a star (hereafter referred to as "the program star"). The placement of the program star is very suggestive of a wind blown cavity in the PN. The question of whether this star actually is interacting with the nebula, or merely along the line-of-sight, is addressed here for the first time.

Photoelectric and CCD spectrographic observations have been made of the star in order to determine its spectroscopic parallax. From the spectra it is determined to be a G2 star (Morgan et al. 1943; Keenan \& McNeil 1976). The Sr II $\lambda 4077$ and $\lambda 4215$ lines reveal that it is a dwarf. In fact, the program star line ratios almost identically match those of HD 224930, which is classified as G2 V (Griffin et al. 1994). The spectrographic observations are confirmed by photoelectric observations which have yielded $a(B-V)_{0}=0.65$, a value consistent with a spectral type of G3 V.

Adopting an absolute visual magnitude corresponding to the spectral and luminosity classification gives a spectroscopic parallax of about $480 \pm 30 \mathrm{pc}$. This result agrees quite well with the accepted distance to the nebula. Minkowski (1959) used spectroscopic parallax of the late-type companion of the central star to calculate a distance of $430 \pm 60 \mathrm{pc}$. More recently, Pottasch (1996) derives a distance of about $500 \mathrm{pc}$. Within the error limits, NGC 246 and the program star are at the same distance. The possibility of an interaction between the two, therefore, cannot be excluded. Higher resolution imaging, and kinematical information is needed of NGC 246 in order to confirm whether an interaction actually is occurring between the PN and the program star. If an interaction is taking place it would provide an excellent laboratory for testing new models of wind-PN interactions. Since the program star is a G2 V, it might also yield important insights into the predicted heliopause.

\section{REFERENCES}

Griffin, R. F., Yoss, K. M., \& Miller, G. J. 1994, The Observatory, 114, 45

Keenan, P. C., \& McNeil, R. C. 1976, An Atlas of Spectra of the Cooler Stars: Types G, K, M, S, and $C$ (Columbus, $\mathrm{OH}$ :Ohio State University Press)

Minkowski, R. L. 1959, Annual Report of the Director, Mt. Wilson and Palomar Observatories, 1959-60, p. 18

Morgan, W. W., Keenan, P. C., \& Kellman, E. 1943, An Atlas of Stellar Spectra for Spectral

Classification (Chicago:University of Chicago)

Pottasch, S. R. 1996 A\&A, 307, 561 